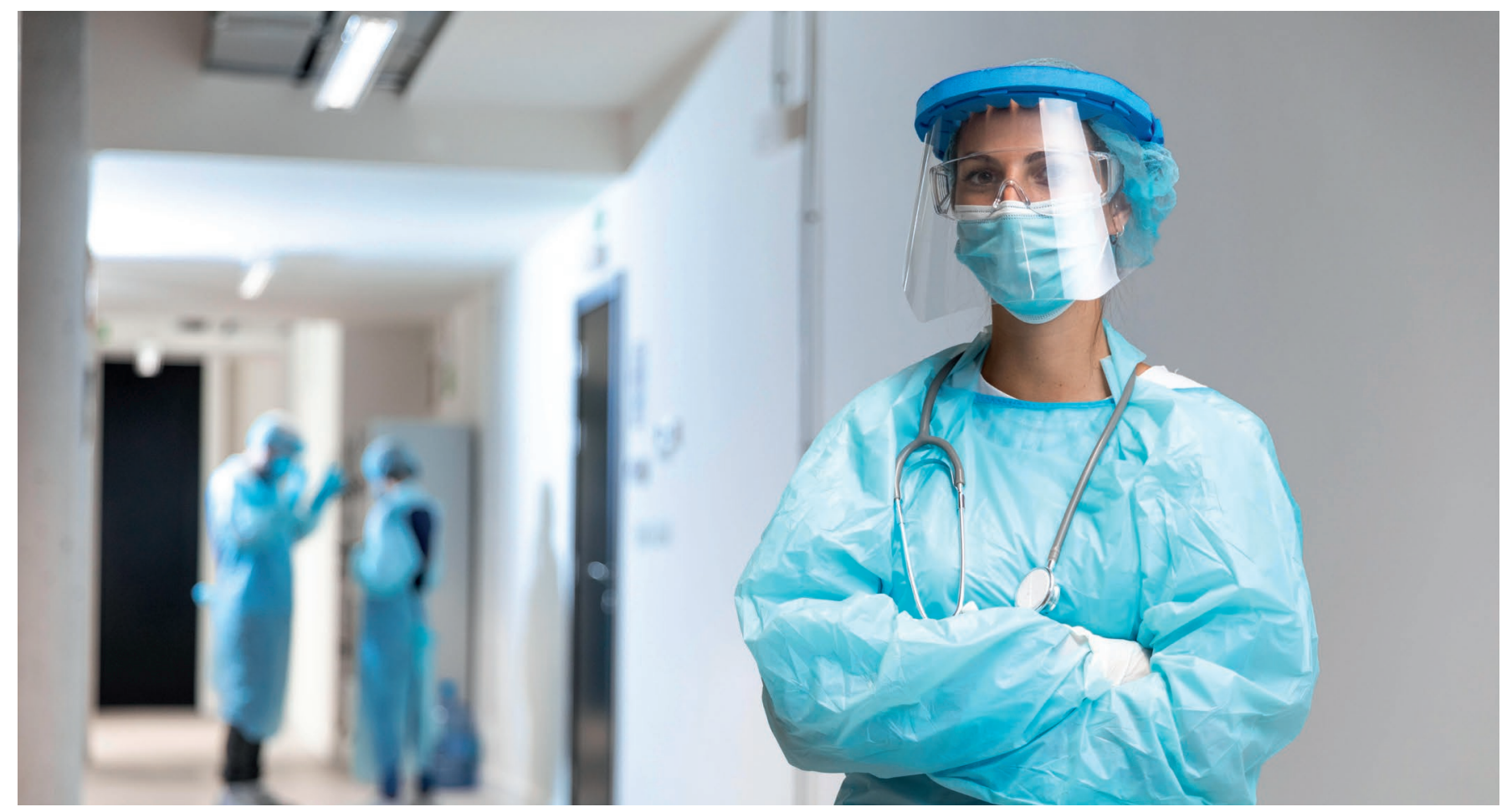

\title{
Was benötigt Intensivpersonal in der Krise?
}

Wenn die Belastung für das Intensivpersonal zu groß wird, kann das ernsthafte Folgen für deren Gesundheit haben. Deshalb ist es wichtig, frühzeitig die Ursachen zu erkennen und Lösungsansätze anzubieten. Gerade die COVID-19-Pandemie hat noch einmal mehr aufgezeigt, wie wichtig der psychosoziale Support von Teams ist.

$\mathrm{E}$ ine Krise bezeichnet häufig einen Wendepunkt einer kritischen Situation, die sich entweder zur Katastrophe entwickelt oder zur Aufdeckung von Chancen führen kann. Eine Zuspitzung zur Krise wurde im Gesundheitswesen in der vergangenen Pandemie regelmäßig dann konstatiert, wenn erforderliche Kapazitäten der Intensivmedizin knapper dargestellt wurden und Belastungen daraus abgeleitet worden sind.

\section{WELCHE BELASTUNGEN WAREN RELEVANT?}

Psychische und physische Herausforderungen sind in der Intensiv- und Notfallmedizin sowohl innerklinisch als auch außerklinisch Alltag. Eine globale Pandemie fordert das gesamte nationale Gesundheitssystem bis an die Belastungsgrenzen, darin im Speziellen auch deren Mitarbeiter*innen in der Patient*innenversorgung. Werden darüber hinaus vermeidbare Belastungsfaktoren angetriggert, kann das zur Überforderung von Personal und damit folglich der Intensivmedizin selbst führen. 1, 2,3,4

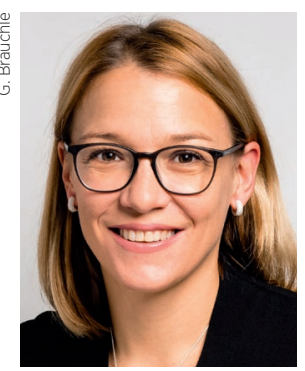

Von

Maria Brauchle

Akademisch zertifizierte Expertin in der Intensivpflege, Abteilung für Anästhesie und Intensivmedizin am Landeskrankenhaus Feldkirch

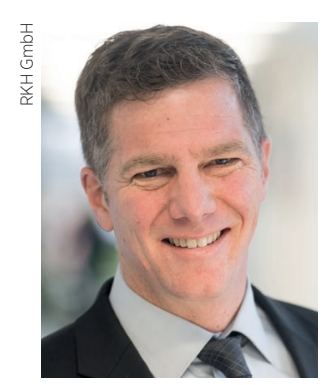

Tilmann MüllerWolff, M.A.

Pflege- und Gesundheitswissenschaftler, Akademieleitung Regionale Kliniken Holding RKH GmbH, Ludwigsburg

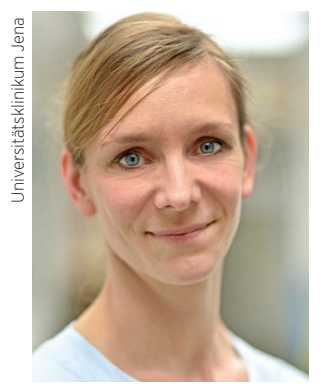

und

Dr. Teresa Deffner Dipl.-Rehapsych. (FH), Psychologin Operative Intensivstationen und Pädiatrische Intensivstation, Klinik für Anästhesiologie und Intensivmedizin Kinderklinik, Sektion Neonatologie und Pädiatrische Intensivmedizin, Universitätsklinikum Jena
Über ein Jahr Patient*innenversorgung während der Pandemie hat gezeigt, dass Mitarbeiter*innen von Intensivstationen, insbesondere wenn sie direkt in die Versorgung von COVID19-Patient*innen eingebunden sind, über eine höhere psychische, aber auch physische Belastung berichten ${ }^{5,6}$ und viele Pflegekräfte ihren Beruf unter den aktuellen Bedingungen nicht mehr ausüben wollen.?

Medizinische Fachgesellschaften haben themenbezogene Ad-hoc-Empfehlungen publiziert, um zusätzlich auf die Herausforderungen hinzuweisen. Träger*innen und Betreiber*innen der Gesundheitseinrichtungen und von Fachgesellschaften haben spontan und reaktiv wohlmeinende Angebote zur Entlastung oder zumindest Unterstützung etabliert, viele davon aus der Not heraus, neuartig und bislang wenig evaluiert. Um diese Angebote in sowohl Nachhaltigkeit als auch Wirksamkeit zu überführen, sind evidenzbasierte, strukturierende und schlussendlich auch evaluierende Schritte notwendig. ${ }^{8}$ 
Mittlerweile ist bekannt, dass u. a. ein Überforderungserleben hinsichtlich der fachlichen Anforderungen ${ }^{9,10}$, aber auch das Fehlen qualifizierten Personals" ${ }^{11}$, die Sorge um die eigene Sicherheit sowie fehlende Schutzsausrüstung ${ }^{11}$ und überdurchschnittliche psychische Belastungen ${ }^{12}$ ausschlaggebend für das Belastungserleben insgesamt sind.

Auch wurde deutlich, dass eine innerklinisch konzertierte Informationsstrategie $^{13}$ ebenso wie wahrgenommene institutionelle und soziale Unterstützung vor psychischer Belastung des Personals schützen ${ }^{14}$, wobei insgesamt die noch fehlende Evidenz für wirksame Maßnahmen zu beklagen ist ${ }^{13}$.

Zudem kamen auf das medizinische Personal massive und sehr kurzfristige fachliche Herausforderungen zu, die sich aus der Intensität einer ersten und zweiten Pandemiewelle und dem entsprechendem Patient*innenzustrom ergaben ${ }^{\text {vgl.15, } 16,17}$ Die hohe mediale Präsenz der Pandemie, voran bestehende Belastungen bei insbesondere der Pflege in der Intensivmedizin ${ }^{18}$, ein gänzlich neues Erkrankungsbild mit hohen Patient*innenzahlen und lediglich geringfügig ausgeprägte Kriseninterventionskompetenzen im Gesundheitssystem waren in Kombination die Verstärker einer Krise beim Intensivpersonal.

\section{WELCHE KONKRETEN EMPFEHLUNGEN KÖNNEN HERANGEZOGEN WERDEN?}

Hilfreich ist es, in Bereichen nach etablierten Lösungen zu suchen, die sich bereits länger damit befasst haben. International gibt es bereits Empfehlungen für die psychosoziale Unterstützung in allgemeinen Notfallsituationen., 19 Auch für Einsatzkräfte in der Präklinik liegen seit 2010 Qualitätsstandards und Leitlinien für die psychosoziale Notfallversorgung (PSNV) nach psychisch extrem belastenden Ereignissen vor.

Für das innerklinische Setting finden sich kaum flächendeckende, strukturierte und standardisierte Angebote einer PSNV für Betroffene (Angehörige) und Beschäftigte in krisenhaften und potenziell traumatisierenden Situationen. ${ }^{20}$ Dabei bleibt häufig unberücksichtigt, dass medizinisches Personal neben den eigenen Belastungen auch diejenigen der
Betroffenen (Angehörige, Patient*innen) miterlebt. Beispielsweise konnten Angehörige pandemiebedingt monatelang ihre schwerst erkrankten Familienmitglieder nicht auf der Intensivstation besuchen. Telefon, Live-Videotechnik oder andere Kommunikationsmittel mussten improvisiert eingesetzt werden, um Hygieneschutz und Präventionsmaßstäben gerecht zu werden.

Einzig die Deutsche Interdisziplinäre Vereinigung für Intensiv- und Notfallmedizin (DIVI) veröffentlichte bereits im März 2020 Handlungsempfehlungen für die „Klinische psychosoziale Notfallversorgung im Rahmen von COVID-19“.20 Die hierin aufgeführten Handlungsempfehlungen könnten einen ersten Hinweis zur Nutzung auch über die Pandemie hinaus aufzeigen.

Konkret wird die Einrichtung eines Themenschwerpunkts „Klinische psychosoziale Notfallversorgung“ im Rahmen der bereits eingerichteten Krisenstäbe empfohlen. Hieran zu beteiligen sind das verfügbare psychosoziale Fachpersonal bzw. Leitungspersonen (wie etwa Klinikseelsorge, psychosoziale kollegiale Peers, innerklinische Krisenintervention, psychologische, psychotherapeutische, psychiatrische, psychosomatische Versorger*innen, palliativmedizinischer Dienst, arbeitsmedizinischer Dienst, betriebliches Gesundheitsmanagement). Diese sollen an der Etablierung eines Notfallversorgungskonzeptes und dessen Integration in klinische Pandemieund Notfallpläne beteiligt sein. Hieraus können dann zielgruppenadaptierte Maßnahmen abgeleitet werden. Ziele solcher PSNV sind die psychische Stabilisierung in der Situation, Unterstützung konstruktiver Bewältigungsstrategien, Gewährung von adäquaten Hilfen und Weitervermittlung in dieselben, Vermeidung von psychosozialen Belastungsfolgen und Entlastung des klinischen medizinischen Personals. ${ }^{20}$

Das Intensivpersonal ist während der vergangenen Monate der Pandemie zum Sinnbild herausragender Leistungen unter hoher Belastung stilisiert worden. Dabei sind gerade die Intensivpflegekräfte und intensivmedizinisch tätigen Ärzt*innen diejenigen, die auf Belastungsspitzen aufgrund von Notfallsituationen durch teilweise jahreslange Ausbildung und Training vorbereitet wurden. Die souveräne Jonglage zwischen physischen, psychischen, emotionalen und fachlichen Herausforderungen der Tätigkeit auf einer Intensivstation ist ebendort üblicher Alltag. ${ }^{21}$ Lediglich die Summe und Dauer der Belastungen stiegen durch die globale Pandemie und deren fehlende Einschätzbarkeit extrem rasch und umfangreich an. Die bereits zitier-

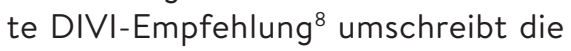
herausfordernden Belastungen für das Intensivpersonal wie folgt:

- Ressourcenengpässe (personell, materiell etc.) können als außergewöhnliche Belastung wahrgenommen werden sowie Gefühle des Ausgeliefertseins hervorrufen.

- Fachliche Überforderung (professionelles Hilflosigkeitserleben), vor allem bei Mitarbeiter*innen, die kurzfristig in intensivmedizinische, intensivpflegerische und notfallmedizinische Tätigkeiten eingearbeitet werden.

- Geringe Kontrolle über das Geschehen - keine Beeinflussung, wann wie viele Patient*innen auf Intensivstationen verlegt werden.

- Gesteigerte Erwartungshaltung und hoher Druck durch Medien (Idealisierung der Tätigkeit von Intensivpersonal).

- Körperliche Belastungen und Erschöpfung aufgrund des kontinuierlichen Tragens der persönlichen Schutzausrüstung bei Tätigkeiten an isolierten Patient*innen.

- Vermehrte Konfrontation mit psychischen Extrembelastungen (kritisch) kranker Patient*innen und deren Angehörige (auch telefonisch/ bei Videotelefonie) einerseits durch ein erhöhtes Patient*innenaufkommen, andererseits durch umfassende Besuchsverbote.

- Psychische Belastungen des Personals durch ggf. notwendige Triage/ Sichtung von Patient*innen bei begrenzten oder fehlenden materiellen Ressourcen und dadurch wiederholte Gefährdung durch extremes Hilflosigkeitserleben.

- Sorgen um die eigene Gesundheit und die der eigenen Familie, da die Infektionsgefahr durch die berufliche Exposition deutlich gegenüber der Normalbevölkerung erhöht ist. 
- Gesteigerte Herausforderungen durch die Doppelbelastung der Alltagsorganisation inklusive der familiären Aufgaben und zugleich durch die deutlich intensivierten beruflichen Belastungen.

Im Weiteren empfiehlt die DIVI, Maßnahmen der Primär-, Sekundär- und Tertiärprävention einzusetzen. Hierbei orientiert sich die Empfehlung an publizierten Erkenntnissen aus den Bereichen der Gesundheitswissenschaften und Präventionsforschung sowie Erfahrungen aus der allgemeinen Kriseninterventionsarbeit. Die konkrete Umsetzung wird den Einrichtungen und deren Möglichkeiten überlassen. Primärpräventiv könnten regelmäßige Teambriefings mit transparenten Informationen zur Pandemie und Gefahrenlage ebenso sein wie konkrete fachliche Trainings zur Beherrschung der neuen Krankheitsbilder und Handlungsweisen. Sekundärpräventiv können Maßnahmen etabliert werden, die frühzeitiges Erkennen von Belastungsfolgen bei Mitarbeiter*innen, die Einrichtung von Kompensationsangeboten und die Information hierüber unterstützen. Als Tertiärprävention wird das frühzeitige Berücksichtigen von etwaigen Folgeangeboten (z. B. Therapien oder Beratungsangebote) für betroffenes Personal aufgeführt.

\section{WAS KÖNNEN WIR BESSER MACHEN?}

Um die Erfahrungen der beschriebenen und vergangenen Krise konstruktiv und präventiv nutzbar zu machen, sollten einige darin entdeckte widrige Umstände als Chance zur Verbesserung betrachtet werden.

Wirksame Strategien erfordern umfassendere Erforschung im Bereich der Verbesserung der Arbeitsbedingungen von Klinikmitarbeiter*innen. Auch zur Wirksamkeit von Krisenintervention und deren häufig angewandten Methoden besteht noch weiterer Untersuchungsbedarf. Die Anwendung von Kriseninterventionstechniken aus anderen Settings und Zusammenhängen für in der Klinik beobachtete Phänomene und Situationen wirkt derzeit noch weitestgehend improvisiert. Zukünftig sollten diese Interventionen folglich strukturiert angewendet und wirksam-

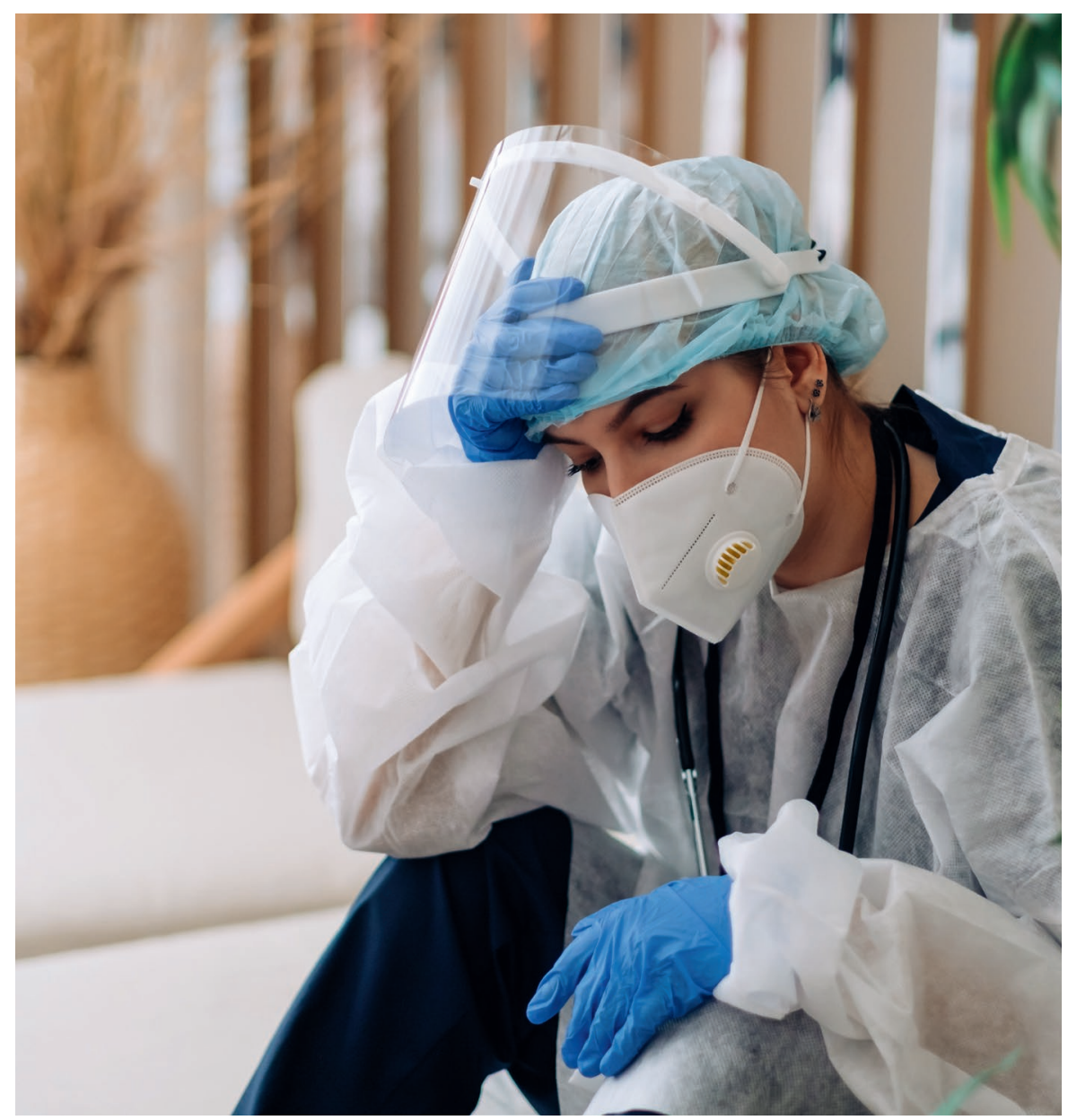

keitsorientiert evaluiert werden. Zudem sollte in Zukunft noch viel deutlicher in die frühzeitige Bildung von Krisen- und sonstigen Coping-Strategien bei Aus-, Fort- und Weiterbildung von Gesundheitsberufen investiert werden. Je besser also die Vorbereitung, desto höher ist vermutlich die Krisenresilienz, wenn es darauf ankommt.

\section{Referenzen:}

Lai J, Ma S, Wang Y et al. JAMA Netw Open. 2020 Mar 2;3(3):e203976

2 North Atlantic Treaty Organization - NATO. Psychosocial Care for people affected by disasters and major incidents. A model for designing, delivering and managing psychosocial services for people involved in major incidents, conflict, disasters and terrorism. 2008

3 Butollo W, Karl R, Krüsmann M. Reihe Forschung im Bevölkerungsschutz, 2012, Bd. 8, Bundesamt für Bevölkerungsschutz und Katastrophenhilfe, Bonn

4 Scelsi S, European federation of Critical Care Nursing associations. Letter to the European nursing colleagues. aniarti/efccna, 2020 Mar 08

5 Bohlken J, Schömig F, Lemke MR, Pumberger M, Riedel-Heller SG. Psychiatr Prax. 2020 May; 47(4):190-197

6 Danet Danet A. Med Clin (Engl Ed). 2021 May 7;156(9):449-458

7 https://www.aerzteblatt.de/nachrichten/123219/ Ein-Drittel-der-Intensivpflegenden-will-Arbeitsstelle-aufgeben

8 Arndt DA, Heininger S, Katharina S, Hinzmann D, Walcher F, Brauchle M, Müller-Wolff T. (2020). In: ErgoMed - Praktische Arbeitsmedizin
9 Leng M, Wei L, Shi X, Cao G, Wei Y, Xu H, Zhang X, Zhang W, Xing S, Wei H. Nurs Crit Care. 2021 Mar 26(2):94-101

10 Caillet A, Coste C, Sanchez R, Allaouchiche B. Anaesth Crit Care Pain Med. 2020 Dec;39(6):717722

11 Fernandez R, Lord H, Halcomb E, Moxham L, Middleton R, Alananzeh I, Ellwood L. Int J Nurs Stud. 2020 Nov; 111:103637

12 Lapum J, Nguyen M, Fredericks S, Lai S, McShane J. Can J Nurs Res. 2021 Mar; 53(1):5-15

13 Pollock A, Campbell P, Cheyne J, Cowie J, Davis B, McCallum J, McGill K, Elders A, Hagen S, McClurg D, Torrens C, Maxwell M. Cochrane Database Syst Rev. 2020 Nov 5;11:CD013779

14 Labrague LJ, De Los Santos JAA. J Nurs Manag. 2020 Oct;28(7):1653-1661

15 Müller-Wolff T, van den Hooven T. Pflegewissen schaft 2. Sonderausgabe: Die Corona-Pandemie; S 64-65; 05.2020, ISSN 1662-3029; hps media Hungen

16 Schindele D, Müller-Wolff T. Pflegewissenschaft 2. Sonderausgabe: Die Corona-Pandemie; S 6-8; 2020, ISSN 1662-3029, hps media, Hungen.

17 Shores R, Fowler K, McDonough J, Suralis A, Scindele D, \& Müller-Wolff T (2021). Anesthesia EJournal, 8(2), 27-31

18 Müller-Wolff T, Ullrich L, van den Hooven T et al. Deutscher Ärzteverlag, Deutsche Interdisziplinäre Vereinigung für Intensiv- und Notfallmedizin 2019; 10 (3)

19 Inter-Agency Standing Committee (IASC) (2007). IASC Guidelines on Mental Health and Psychosocial Support in Emergency Settings. Geneva: IASC

20 Deffner T, Hierundar A, Arndt D, Hinzmann D. 2020 https://www.divi.de/empfehlungen/publika tionen/viewdocument/3429/covid19-psychosoziale-notfallversorgung Abgerufen am 6.6.2021

21 Müller-Wolff T. In: Deffner T, Janssens U, Strauß $B$ (Hrsg.): Praxisbuch Psychologie in der Intensivund Notfallmedizin, 2021, Verlag Medizinische Wissenschaft, Berlin 